\title{
Sentiment Research on Student Feedback to Improve Experiences in Blended Learning Environments
}

\author{
R.K. Kavitha
}

\begin{abstract}
Educational data mining has aroused a great research interest among the educational institutions nowadays. Blended learning is used as a pedagogy in the field of teaching and learning. Blended learning is a fusion of online digital media with conventional teaching in classrooms where the teacher and student has to be present physically throughout teaching duration. Teacher-student interaction is made possible using the internet during the non-contact hours. For rendering a valuable blended learning environment, it is essential to gather users' opinion or feedback on this learning methodology. Therefore, opinion-mining techniques have been used in this paper for helping the academicians to improve and promote such learning environments. Students positive or negative feelings towards the subject teaching can be analyzed using these techniques. This paper discusses how sentiment analysis can be performed on the feedback collected in a learning management system with the intention of advancing the teaching learning process. This work presents the experimental results that were obtained after comparison of various feature selection methods say Chi-square, Information Gain, Mutual Information and Symmetrical Uncertainty.
\end{abstract}

Keywords-Feature Selection, Blended learning, Text Classification, Sentiment Analysis, Opinion Mining.

\section{INTRODUCTION}

Educational Data Mining (EDM) helps to deal with problems in the field of education. Textual data appears in a variety of forms like tweets, emails, chats, movie reviews, product reviews etc. To extract useful information from textual data, a technique called sentiment mining can be used. The main task of sentiment mining technique is to classify text that has diversified applications [8]. Handling huge textual data nowadays has raised the need of text classification [16]. Analyzing student feedback helps the teacher to improve the teaching quality. Student feedback for the course was collected through the Learning Management System (LMS) that provided a convenient way for the students to convey their feedback in the form of free text. The proposed work discusses the advantages of using Sentiment Analysis on student's feedback. This method categorizes the feedback as positive, neutral or negative.

Feature selection methods when used in classifying data available in text format results in dimensionality reduction of the feature space. This high dimensionality feature space reduction is achieved by recognizing informative features using feature selection [3]. Advantages of feature selection are listed as follows: a) classification performance

Revised Manuscript Received on September 10, 2019.

R.K. Kavitha, Assistant Professor(SRG), Department of Computer India.

(E-mail: kavitha.rk.mca@kct.ac.in) Applications, Kumaraguru College of Technology, Coimbatore, Tamilnadu,

improvement b) classification model construction and testing speed up and c) Model interpretability. This paper presents the results of applying various metrics of feature selection technique on the data set. Chi-squared, Information gain, Symmetrical Uncertainty and Mutual Information were applied on the collected data. Similarly, among the available sentiment analysis techniques, only few techniques like Naive Bayes (NB), Support Vector Machines (SVM) and Max Entropy (MaxEnt) were observed to provide better performance.

\section{SURVEY OF LITERATURE}

In literature, numerous studies has been reported which used various document text classification techniques and metrics. Soundness of these techniques has also been presented [16]. Quite a few studies in the literature are based on feature selection method, which focuses on performance in statistical learning [2]. Methods like Chi-square, Information Gain (IG), Mutual Information (MI) etc. are employed to improve the process of retrieving information on the collected dataset [4]. Earlier studies have proved that these methods were more effective compared to others [7]. An inverse relation between the parameter correction and feature reduction was observed in a study [4]. In addition, a research concluded that feature selection depending on unusual ratio has more advantage when compared to naïve Bayes technique. Also, support vector machines (SVM) prediction technique exhibits an improved result [7]. From the literature results, it was decided to perform feature selection for classifying the text data to display a precise accuracy rate.

\section{SENTIMENT ANALYSIS}

In order to discover and mine subjective information from source data, techniques like natural language processing, text analytics and computational linguistics were used [8]. Victory of a method can be decided by monitoring features that the users like or dislike. This can be done using feature extraction technique. Normally polarity of the sentiment can be classified as positive, neutral or negative. Also, this technique can be used to mine various user emotions like joy, sad, love etc. from the text data. Words can have optimistic meaning in some ways and pessimistic in other. In the field of education, it will be great use in understanding the feelings or opinion of students undergoing a course. One way to understand the emotions of e-learners

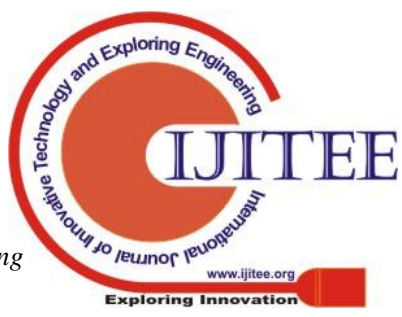


is through the textual comments and discussions shared by them. It was observed from the literature that most of the studies focused on cognition of the e-learners while the emotion or sentiment were less studied. With the intention of improving the teaching quality, it was decided to analyze the feedback of the learners in this research work.

The student feedback collected through LMS is further stored in a database which may contain thousands of textual feedback for the various courses handled by the faculty. Applying sentiment analysis to student feedback aims to determine the overall contextual polarity of it. For sentiment classification, the feedback is searched for sentiment bearing words like adjectives and domain specific technical words. With the help of lexical resources, the words can be classified as positive and negative. This detail comes handy for the subject handling staff, thus enabling them to handle the classes more effectively.

Feature selection, which is a machine learning technique, works by selecting subsets of pertinent features obtained from a large data set. While using this method, the output quality is thus not compromised [3]. Feature selection algorithms can be applied in various areas like image processing, bioinformatics, banking, text categorization, etc. The key idea behind this technique is the clear discrimination of individual features. The basic steps involved in feature selection are depicted in Figure 1. The steps used to identify features are generation of subset, evaluation of subset, stopping criterion, and validation of results[10].

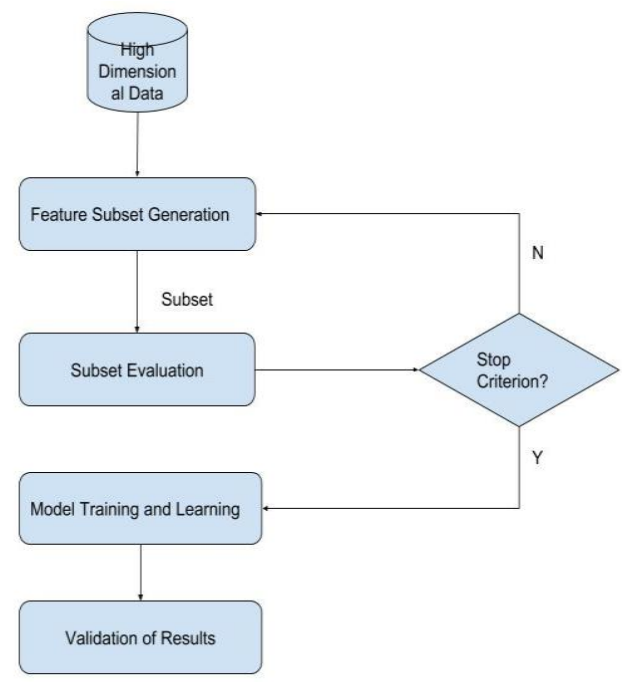

Fig. 1 - Feature Selection Steps

Various feature selection methods are as given below:

\section{1) Chi-square statistics (CHI):}

The statistical Chi-Square test of independence helps to find the noteworthy relationship between two categorical variables. This test will examine the significant difference in the observed and expected frequencies of the variables under consideration. The formula for calculating Chi-Square statistic is expressed as follows:

Chi-squared $=\sum$ (observed-expected $)^{2} /($ expected $)$

Expected $=($ Row Total X Column Total $) /$ Overall Total

\section{A. Feature Selection Methods}

\section{$\mathrm{DF}=($ Rows- 1$) \mathrm{X}$ (Columns-1)}

Chi-square technique thus measures the independence among the term ' $t$ ' and class ' $c$ '. Similarity in the distribution will be calculated using one degree of freedom. The formula used to calculate the term similarity is as given below:

$$
\mathrm{X}_{(\mathrm{t}, \mathrm{c})}^{2}=\mathrm{D} \times(\mathrm{PE}-\mathrm{MQ})^{2} /(\mathrm{P}+\mathrm{M}) \times(\mathrm{Q}+\mathrm{N}) \times(\mathrm{P}+\mathrm{Q}) \mathrm{x}
$$

$(\mathrm{M}+\mathrm{N})$

where $\mathrm{D}=$ total no. of documents

$\mathrm{P}=$ no. of documents belonging to class $\mathrm{c}$

containing term $\mathrm{t}$

$\mathrm{Q}=$ no. of documents having $\mathrm{t}$ without $\mathrm{c}$

$\mathrm{M}=$ no. of documents of class $\mathrm{c}$ without $\mathrm{t}$

$\mathrm{N}=$ no. of documents of any class without $\mathrm{t}$

Chi-square statistic test is basically for association which is a non-parametric test. Therefore, it can be used for nominal data too. It is mainly used in tabular association analysis.

\section{2)Information gain ( $I G)$ :}

IG which is called as the gain ratio and entropy reduction is measured using this. Category prediction can be achieved with the help of the information about existence or nonexistence of a term or an attribute in the document under consideration. It is used to measure goodness of a term in machine learning field. Information gain helps to identify the attribute from a given set of training feature vectors that will be able to discriminate the classes that are to be learned. It also says the level of significance of the attribute in a feature vector. This technique will be used to decide the ordering of attributes in the decision tree nodes.

\section{3)Mutual Information (MI):}

Technique used for computing the correlation of knowledge between two attributes is called as Mutual information (MI). It is used to find the dissimilarity between amount of marginal entropy and combined entropy. Mutual information constantly tends to be zero for any two totally independent objects. The maximum dependency state based on mutual information helps for selecting the features. This work uses the mutual information technique with Shannon's entropy [2].

A high dimensional data is expressed as $\mathrm{D}=\mathrm{N} \times \mathrm{M}$, where $\mathrm{M}$ is the no. of features and $\mathrm{N}$ is the no. of the instances. Consider two features that are random, say $\mathrm{x}$ and $\mathrm{y}$ whose probability density is given by $(\mathrm{x})$ and $\mathrm{p}(\mathrm{y})$ and whose joint probability density function is denoted as $\mathrm{p}(\mathrm{x}$, $\mathrm{y})$. Then the mutual information (MI) can be expressed as follows [4]:

$\mathrm{MI}(\mathrm{X}, \mathrm{Y})=\mathrm{H}(\mathrm{X})-\mathrm{H}(\mathrm{X}, \mathrm{Y})$

$\mathrm{MI}(\mathrm{X}, \mathrm{Y})=\mathrm{H}(\mathrm{X}, \mathrm{Y})-\mathrm{H}(\mathrm{X} \mid \mathrm{Y})-\mathrm{H}(\mathrm{Y} \mid \mathrm{X})$

Normally, the feature values are observed to be discrete in nature. Marginal entropies are referred to as $\mathrm{H}(\mathrm{X})$ and $(\mathrm{Y})$, conditional entropies as $\mathrm{H}(\mathrm{X} \mid \mathrm{Y})$ and $\mathrm{H}(\mathrm{Y} \mid \mathrm{X})$, joint entropy of $\mathrm{X}, \mathrm{Y}$ is denoted as $\mathrm{H}(\mathrm{X}, \mathrm{Y})$. Thus, the mutual information computed based on $\mathrm{Y}$ is found equal to the quantity of it after studying $X$. Hence, it is observed that calculation for $\mathrm{X}$ and $\mathrm{Y}$ leaves the measurement unchanged.

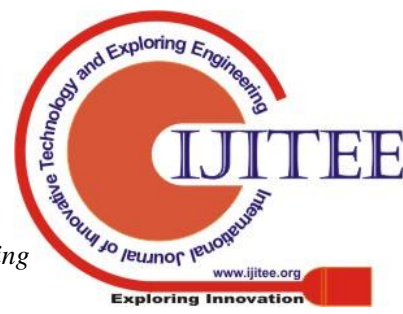


In feature selection technique, it is observed the excellent features in the data set may enhance the mutual information $\mathrm{MI}(\mathrm{X}, \mathrm{Y})$ to a maximum value considering $\mathrm{X}$ as the feature vector and $\mathrm{Y}$ as the class indicator. The symmetric uncertainty (SU) [1] is extended from mutual information method after normalizing to feature entropy value and feature entropy values with a class label. SU helps to find the feature effectiveness that are used for data classification by researchers [4]. The proposed work also uses SU to find the correlation among two features or between a feature and a class value.

\section{4)Symmetric Uncertainty ( $S U)$ :}

The fitness of features of symmetric uncertainty method can be calculated by finding the difference between feature and the target class. The feature with high SU value gets more weightage. Symmetric uncertainty defined as

$\mathrm{SU}(\mathrm{X}, \mathrm{Y})=(2 \mathrm{x} \mathrm{MI}(\mathrm{X}, \mathrm{Y})) / \mathrm{H}(\mathrm{X})+\mathrm{H}(\mathrm{Y})$

In the above expression, $\mathrm{H}(\mathrm{X})$ denotes the entropy value of discrete random variable $\mathrm{X}$. A value 1 for $\mathrm{SU}(\mathrm{X}, \mathrm{Y})$ specify that object knowledge value is strong and value 0 for it indicates the independence of $\mathrm{X}$ and $\mathrm{Y}$. Thus the SU coefficient can be used to control redundancy and increase the classification accuracy.

\section{B. Classification Techniques}

Classification, a popular data mining technique, allocates items to target classes thus providing accurate prediction of the target class in each case for the given data.

\section{1)Decision Tree:}

Decision tree classification approach is most helpful in classification problems. Itis a simple support tool used for decision making and uses a tree-like model of outcome variables. The flow chart like tree structure is constructed in a top down fashion using a recursive, divide and conquer style. Algorithms like ID3, C4.5, Classification and Regression Trees are used to classify data sets. A top-down approach is followed in this method that starts with a training set of data with associated class labels.

\section{2)Support Vector Machine (SVM):}

As a well-liked and useful method for data classification, SVM is used to classify linear and nonlinear data. The objective here is to develop a model in order to predict target value of data occurrence in a given test data set. The classification goal in SVM is to divide two classes with a function and construct a classifier which work fine on an unseen data. One of the simplest form of classifier which helps to solve fundamental classification problem is called as maximal margin classifier. Slack variables helps in tolerating training errors thus decreasing the noise in training data.

\section{3)Nä̈ve Bayes Classifier:}

The foundation of Naive Bayes, a fundamental probabilistic classifier is the Bayes theorem. This is widely used to classify text and is applied in areas such as spam detection in e-mails, document categorization, language detection and sentiment detection. According to Bayes theorem,

$$
\text { Posterior }=\frac{\text { Prior } * \text { likelihood }}{\text { Evidence }}
$$

\section{4)k-NN:}

$k$-NN which is considered as a non-parametric, slow learning algorithm is used in database applications where the data points are divided into several classes to forecast new sample points. This method reduces data in two steps. Having a test document, the first step is to place the $k$ nearest samples in the midst of training documents with the help of a document-document similarity index. The similarity score of each nearby document to the test document is helps to compute the weight of the categories that are pre-assigned to training document. The next step is to calculate the likelihood of each group by summing up the weight of the group of $k$-nearest documents.

\section{PROPOSED STUDY}

The importance of blended learning was understood after observing the difficulty of the students in using study resources and in teaming up with instructors and co learners. The students and instructors had the opportunity to contact each other for a period of three to four hours per week. Blended learning environments comes handy when the students need to work together while they are not collocated. The proposed work used a Learning Management System [LMS] in order to enhance the teaching-learning process of the students and instructors. They were given the provision to express their thoughts, discuss, and provide instant feedback and comments in textual form. The stored text can be extracted in requisite format for sentiment analysis. Polarity of the text can be determined by applying appropriate techniques. The faculty handling the course can quickly review the polarity and can take appropriate action that leads to an improved teaching and learning.

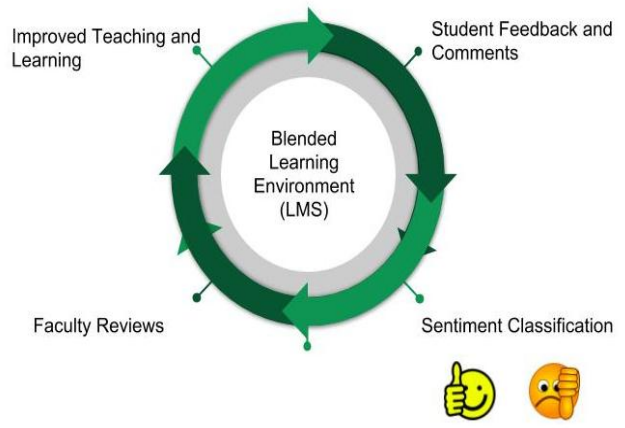

Fig. 2 Research Framework

\section{A. Study Objectives}

1. To analyse student's feedback collected in blended learning environments.

2. To apply and compare the performance of various classification algorithms and feature selection techniques on the collected data set.

\section{B. Methodology of the study}

This section explains about the dataset used in study and the steps used to analyse the various classifier's performance with feature selection metrics.

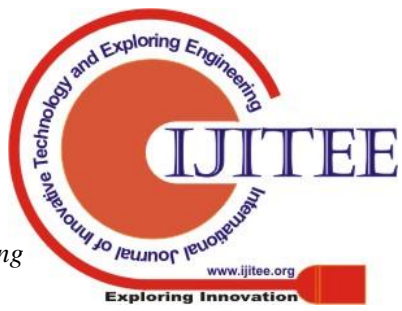




\section{Data collection and Sampling}

Computer science post graduate and undergraduate students were involved in the study. Samples were selected from student group who undertake computer based courses. Roughly two hundred samples were used for the study that was conducted for a full semester of six months duration. The faculty and students keep in touch with each other for around three hours in a week. The course handling faculty used Learning Management System (LMS) for sharing course related materials. Interactions between students and faculty happened with the help of this environment during non-contact hours. Textual information shared in the analysis.

\section{Sentiment Analysis of data}

The textual feedback retrieved from the database experience the following action. Initially the text is spitted in sentences and sentences are further divided into tokens. Space, punctuations and stop words were removed. Stemming was applied. To each token POS (Part Of Speech) tags are attached using Natural Language Tool Kit (NLTK). Subsequently, self-created tags available in the dictionaries are created for this project. These tags have something more to say about the word. Term document matrix was prepared which was further split into subsets for training and testing. The final stage is to implement the basic extraction rules over the text that is tagged using Python API. Libraries like pandas, NLTK and Valence $A$ ware Dictionary and sentiment reasoner (VADER) were used to analyse data. The performance of different classifiers and algorithms were studied and compared.

\section{RESULT AND DISCUSSION}

Study results were found by applying the feature selection technique on the collected data set. Table 1 and Figure 1 describes the analysis results of the four basic classifiers as mentioned under section III on the collected data. The use of different feature selection with the four classifiers were also presented.

Among the four classifiers SVM performs better with an average accuracy rate of $85 \%$. With SVM, it was found that the classification algorithm IG performs better when compared to other algorithms. At the next level, KNN technique yields better results with IG for the given data set. It can be inferred from table 1 that the accuracy rates of MI and SU feature selection methods were less when compared others.

Table I Classification Accuracy Rate Of Algorithms Using Feature Selection

\begin{tabular}{|l|l|l|l|l|}
\hline CA/FS & $\begin{array}{l}\text { Decision } \\
\text { Tree }\end{array}$ & SVM & $\begin{array}{l}\text { Naïve } \\
\text { Bayes }\end{array}$ & KNN \\
\hline CHI & $51 \%$ & $87 \%$ & $63 \%$ & $76 \%$ \\
\hline IG & $65 \%$ & $90 \%$ & $67 \%$ & $84 \%$ \\
\hline MI & $63 \%$ & $81 \%$ & $50 \%$ & $83 \%$ \\
\hline SU & $60 \%$ & $83 \%$ & $52 \%$ & $81 \%$ \\
\hline
\end{tabular}

$* \mathrm{CA}=$ Classification Algorithm, *FS=Feature Selection discussion section ware collected and stored for further

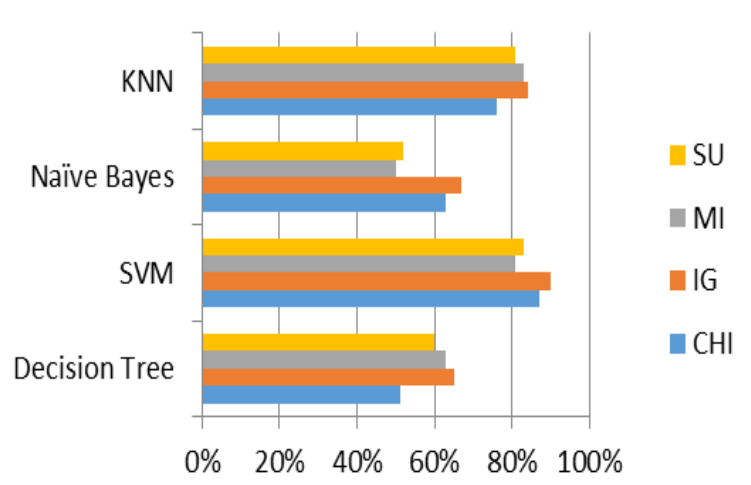

Fig. 3 Comparison of Classification Algorithms

\section{1) Performance measurement and evaluation}

Easiest means to assess classification performance is based on confusion matrix. Finally, the results were evaluated by using precision and recall measures. It was found that the prediction accuracy of student feedback using sentiment analysis technique was quite amenable (Table 2).

Performance of the method discussed in this work was evaluated for precision and recall as shown below.

Correctly extracted textual feedback

Precision $=\quad$ Total textual feedback taken under consideration

Recall $=$ Correctly extracted textual feedback Total relevant textual feedback

From the dataset under study, it was observedthat the feedbacks with positive views were more in number indicating the support for adoption of LMS in near future. A comparison of lessons between the projects and sources was attempted.

Table II Precision And Recall Of Lessons

\begin{tabular}{|l|l|l|l|l|}
\hline \multirow{2}{*}{ FS } & Positive & Negative \\
\cline { 2 - 5 } & Precision & Recall & Precision & Recall \\
\hline SU & 0.50 & 0.59 & 0.60 & 0.66 \\
\hline MI & 0.62 & 0.64 & 0.78 & 0.74 \\
\hline IG & 0.79 & 0.82 & 0.88 & 0.92 \\
\hline CHI & 0.72 & 0.71 & 0.70 & 0.76 \\
\hline
\end{tabular}

As inferred from the table, it can be understood that IG performs the best for sentimental terms selection and exhibits performs well while classifying sentiments during most situations in terms of precision and recall measure. Besides, $\mathrm{CHI}$ is only slightly better than MI.

\section{CONCLUSION}

This paper has described the relevance of applying sentiment analysis on the student feedback, which helps to improve the teaching-learning process. The study has explained the significance of analysing student feedback collected from a Learning Management System very elaborately. It was observed that accuracy of classification achieved with SVM is superior than the other methods. In addition, IG has yielded superior results among the feature 
selection methods. From the study results, it can be inferred that blended learning environments can be made more effective by analysing textual feedback of students. The use of feature selection methods demonstrates improved performance of the classifiers in the field of sentiment analysis.

\section{REFERENCES}

1. Song, Q., Ni, J. and Wang, G. (2013) A Fast ClusteringBased Feature Subset Selection Algorithm for HighDimensional Data. IEEE Transactions on Knowledge and Data Engineering, 25, 1-14.

2. Chow, T.W.S. and Huang, D. (2005) Effective Feature Selection Scheme Using Mutual Information. Neurocomputing, 63, 325-343.

3. Kim, Y., Street, W. and Menczer, F. (2000) Feature Selection for Unsupervised Learning via Evolutionary Search. Proceedings of 6th ACM SIGKDD International Conference on Knowledge Discovery and Data Mining, Washington DC, August, 365-369.

4. Liu, H. and Yu, L. (2005) Toward Integrating Feature Selection Algorithms for Classification and Clustering. IEEE Transactions on Knowledge and Data Engineering, 17, 491-502.

5. Sriram, Bharath, et al. "Short text classification in twitter to improve information filtering. "Proceedings of the 33rd international ACM SIGIR conference on Research and development in information retrieval. ACM, 2010".

6. Aggarwal, Charu C., and ChengXiangZhai. "A survey of text clustering algorithms" Mining Text Data. Springer US, 2012. 77-128.

7. Dasgupta, Anirban, et al. "Feature selection methods for text classification. "Proceedings of the 13th ACM SIGKDD international conference on Knowledge discovery and data mining. ACM, 2007.

8. MagdaliniEirinaki, ShamitaPisal, Japinder Singh, “" Feature -based opinion mining and ranking", Journal of Computer and System Sciences", 2011.

9. Basant Agarwal et al, "Sentiment analysis using common-sense and context information, "Computational Intelligence and Neuroscience”, 2015, pp.01-09.

10. Kintu, M. J., \& Zhu, C. (2016). Student characteristics and learning outcomes in a blended learning environment intervention in a Ugandan University. Electronic Journal of e-Learning, 14(3), 181-195.

11. Nagaraj Balakrishnan, Reshmi S., and R. Arunkumar. "Smart real time rescue system for fishermen." Pak J Biotechnol 15, no. 1 (2018): 73-75.

12. Donnely, R. (2010). Harmonizing technology with interaction in blended problem-based learning. Computers \& Education, 54(2), 350-359.

13. Yeh, Y. C., Huang, L. Y., \&Yeh, Y. L. (2011). Knowledge management in blended learning: Effects on professional development in creativity instruction. Computers \& Education, 56(1), 146-156.

14. SarkaHubackova., Ilona Semradova., (2016). Evaluation of Blended Learning. Procedia - Social and Behavioural Sciences 217, $551-557$.

15. Denker, Katherine J." Student Response Systems and Facilitating the Large Lecture Basic Communication Course: Assessing Engagement and Learning." Communication Teacher 27.1 (2013).

16. Padhy, Neelamadhab, Pragnyaban Mishra, and RasmitaPanigrahi." The Survey of Data Mining Applications and Future Scope." International Journal of Computer Science (2012).

17. Br, Henning, Erik Tews, and Guido Rling." Improving feedback and classroom interaction using mobile phones." Proceedings of Mobile Learning (2005): 55-62.
18. Gauci, Sally A., et al." Promoting student-centered active learning in lectures with a personal response system." Advances in Physiology Education 33.1 (2009): 60-71.

19. Akkoyunlu, B., \&Soylu, M.Y. (2008). A study of student's perceptions in a blended learning environment based on different learning styles. Educational Technology \& Society, 11(1), 183-193. 